\title{
Relato de caso: Schwannoma de origem renal
}

\section{Report case: Schwannoma of renal origin}

Salena Silva Souza ${ }^{1}$. Bruno Hállan Meneses Dias ${ }^{1}$. Alexandre Sabóia Leitão Júnior ${ }^{2}$. Rommel Prata Regadas ${ }^{1}$. João Batista Gadelha de Cerqueira ${ }^{1}$. Ricardo Reges Maia de Oliveira ${ }^{1}$.

1 Universidade Federal do Ceará (UFC), Fortaleza, Ceará, Brasil. 2 Hospital Universitário Walter Cantídio (HUWC), Fortaleza, Ceará, Brasil.

\section{RESUMO}

Schwannomas são tumores que se originam das células de Schawnn. Normalmente se localizam na cabeça, pescoço e membros, mas podem ocorrer em qualquer parte do corpo onde existam nervos mielinizados. O acometimento renal é raro, com poucos casos descritos na literatura. Seu diagnóstico pré-operatório é difícil, visto que se baseiam apenas numa apresentação clínica inespecífica e em características radiológicas limitadas. Embora alguns achados na Ressonância Nuclear Magnética (RNM) possam levantar a suspeição do diagnóstico, esses tumores são frequentemente confundidos com Carcinoma de Células Renais, tendo a ressecção cirúrgica como tratamento padrão. Comumente requerem um diagnóstico patológico. E apesar da maior parte dos casos exibir comportamento benigno, a degeneração maligna pode ocorrer. Nós trazemos o relato de um caso de tumor no hilo renal direito, submetido a ressecção cirúrgica, cujo diagnóstico de Schwannoma renal foi confirmado histologicamente.

Palavras-chave: Neoplasias renais. Neurilemoma. Células de Schwann.

\section{ABSTRACT}

Schwannomas are tumors originated from Schwann cells. Normally is located in the head, neck and limbs, but it can occur anywhere in the body where there are myelinated nerves. Renal involvement is rare, with few cases reported in the literature. The preoperative diagnosis is difficult, since it is based only on a specific clinical presentation and limited radiological resources. Although some MRI findings may raise suspicions for diagnosis, these tumors are often confused with renal cell carcinoma, with surgical resection as the standard treatment. Commonly require a pathological diagnosis. Although most cases exhibit benign behavior, malignant degeneration can occur. We report a case of tumor in the renal hilum, submitted to surgical resection, whose diagnosis of renal Schwannoma was confirmed histologically.

Keywords: Kidney neoplasms. Neurilemmoma. Schwann Cells.

Autor correspondente: Salena Silva Souza, Rua General Silva Júnior, 700/F302, Bairro de Fátima, Fortaleza, Ceará. CEP: 60411-200. Telefone: +55 85 99970-8195. E-mail: salenasouza@hotmail.com

Conflito de interesses: Não há qualquer conflito de interesses por parte de qualquer um dos autores.

Recebido em: 13 Jan 2020; Revisado em: 26 Set 2020; Aceito em: 01 Out 2020. 


\section{INTRODUÇÃO}

Schwannomas são tumores geralmente benignos que se originam da bainha de mielina que envolvem os axônios do sistema nervoso periférico. ${ }^{1}$ São mais encontrados em cabeça, pescoço, membros e mediastino posterior, ${ }^{2}$ mas podem se desenvolver em qualquer parte do corpo. Apenas 3\% de todos os Schwannomas encontram-se no espaço retroperitoneal, e correspondem a $1 \%$ de todos os tumores retroperitoneais. ${ }^{3} \mathrm{O}$ acometimento renal é ainda mais raro, tendo sido descritos menos de 30 casos na literatura. ${ }^{4}$

Schwannomas renais são tumores predominantemente benignos, porém, há relatos de tumores com características de malignidade. Diagnóstico pré-operatório é difícil, devido a dificuldade em diferenciar imagens radiológicas de Schwannomas benignos de tumores malignos. ${ }^{5}$ Nefrectomia radical é escolhida como tratamento na maioria dos casos, sendo feito o diagnóstico frequentemente pelo exame histopatológico da peça cirúrgica.

Nós apresentamos um caso, aprovado pela Comissão de Ética e Pesquisa, de Schwannoma renal tratado pelo serviço de urologia atuante no Hospital Universitário Walter Cantídio.

\section{APRESENTAÇÃO DO CASO}

Paciente, sexo feminino, 49 anos, com queixas de lombalgia leve, principalmente à direita, havia algumas semanas, sem fatores precipitantes. Negava quaisquer sintomas urinários, incluindo hematúria macroscópica. Sem história familiar de câncer geniturinário. Como comorbidade, apresentava hipertensão arterial sistêmica controlada com 2 medicamentos orais. Sem relato de tabagismo. Não havia alterações no exame físico. Exames laboratoriais, incluindo hemograma, função renal, eletrólitos e urinálise, estavam dentro dos padrões de normalidade. Realizou ultrassonografia abdominal que evidenciou uma volumosa lesão sólida na pelve renal direita de 11,2 x 6,8 cm, além de moderada hidronefrose ipsilateral. A partir do resultado deste exame foi solicitada uma tomografia computadorizada que confirmou o achado de volumosa formação expansiva, com captação heterogênea significativa do meio de contraste, em terço médio e superior do rim direito de 10,9 x 8,2cm, ocasionando compressão do sistema coletor e causando hidronefrose (Figura 1). Não foi identificado linfadenopatia nem lesões metastáticas no exame tomográfico.

Devido à natureza sólida e tamanho da lesão, foi indicado nefrectomia radical à direita convencional. Durante cirurgia, notou-se que se tratava de uma lesão tumoral volumosa ( $>$ $10 \mathrm{~cm})$, bem circunscrita, em região anterior do hilo renal, que abaulava pelve e veia cava inferior, sem, contudo, invadir essas estruturas. O intraoperatório transcorreu sem intercorrências, com boa recuperação pós-operatória, recebendo alta hospitalar dois dias após a cirurgia.

No exame anatomopatológico, constatou-se tumoração de 14,0 x 10,0 x $6,0 \mathrm{~cm}$, com cápsula espessada e opaca, de coloração branco-amarelada, apresentando áreas de necrose e degeneração cística. Ao microscópio, havia predominância de células fusiformes, dispostas em feixes, bem como células volumosas, com amplo citoplasma, em meio a vasos de calibre espessado; além de alterações compressivas no parênquima renal adjacente (Figura 2). O laudo da histologia foi de neoplasia fusocelular ricamente vascularizada, sendo sugerido o estudo imunohistoquímico para melhor caracterização da neoplasia. O painel imuno-histoquímico revelou positividade difusa para S-100, além de forte positividade para GFAP (Glial Fibrillary Acid Protein), concluindo o diagnóstico compatível com Schwannoma.

Figura 1. Imagens de tomografia computadorizada mostrando uma massa em hilo renal captante de contraste e com áreas hipodensas centrais.

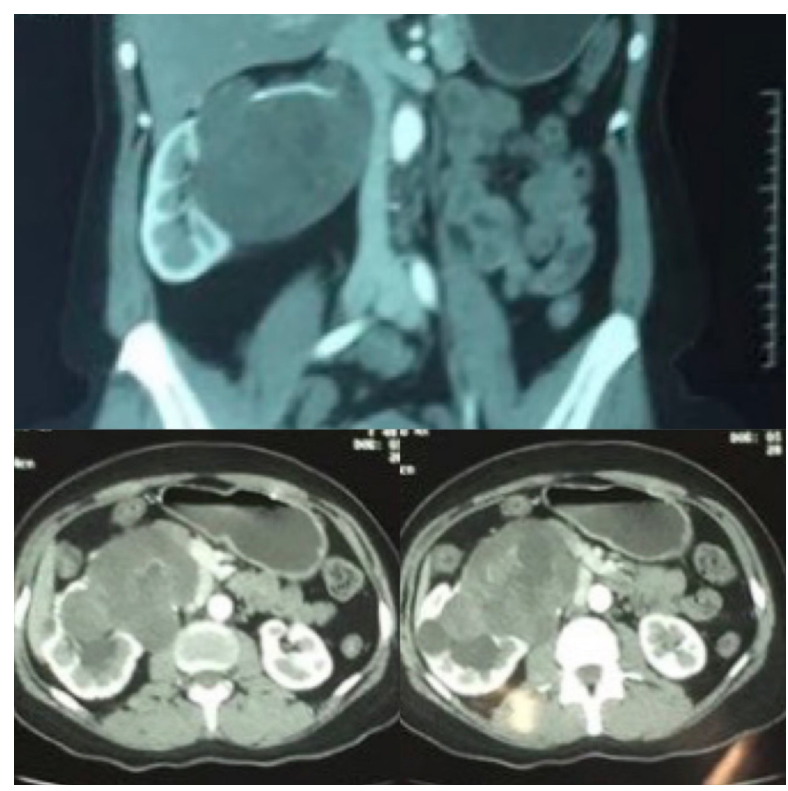

Figura 2. Histologia do Schwannoma renal; A) tumor se separa do parênquima renal por uma cápsula bem formada de fibrose; B) tumor é caracterizado por uma mistura de padrões histológicos de Antoni A e Antoni B.

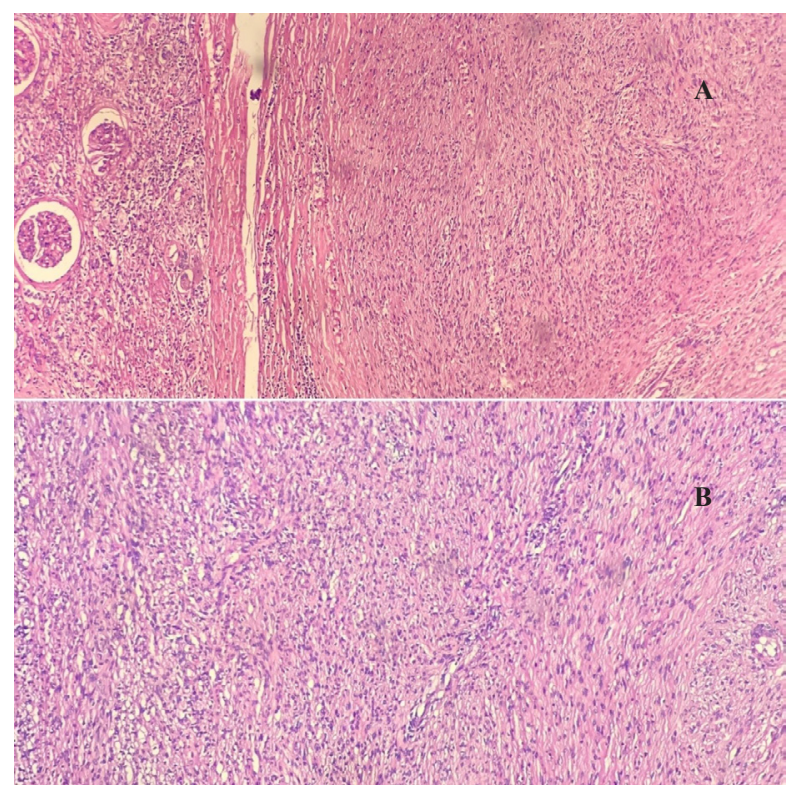




\section{DISCUSSÃO}

Schwannomas são tumores incomuns originados das células de Schwann da bainha dos nervos periféricos. Localizam-se comumente em tecidos subcutâneos de extremidades e de áreas da cabeça e pescoço, mas também podem ocorrer em tecidos moles do mediastino e retroperitôneo. ${ }^{6}$ Schwannomas retroperitoneais constituem apenas $1 \%$ de todos os tumores retroperitoneais, e quando se desenvolvem no retroperitôneo, cerca de 3\% de todos os casos de Schwannoma, usualmente são associados com plexos nervosos lombossacrais e menos comumente crescem de órgãos retroperitoneais como o rim. ${ }^{7}$ Já que se tratam de tumores neurais diferenciados de origem mesenquimal, eles crescem da superfície do seu nervo de origem, e os principais nervos do rim consistem em fibras simpáticas e parassimpáticas que acompanham a artéria renal ao entrar no hilo. O que poderia explicar a frequente localização do Schwannoma no hilo renal. ${ }^{2}$

O primeiro caso de tumor neuronal localizado no hilo do rim foi descrito por Philips e Baumrucker em 1956. ${ }^{8}$ Desde então casos esporádicos de Schwannoma renal têm sido descritos na literatura em variadas línguas, não totalizando 30 casos.

Idade dos pacientes varia de 18 a 84 anos, ${ }^{2,9}$ com uma média em torno de 50 anos e leve predominância pelo sexo feminino. Apresentação clínica costuma ser inespecífica, e devido ao crescimento lento do tumor, a maioria dos pacientes são assintomáticos. Mas há relato de dores em flanco, hematúria e massa palpável.

A massa é frequentemente localizada na parte central do rim, pela maior concentração de tecido nervoso nesse nível, comprimindo o hilo e a pelve renal. ${ }^{2}$ Podendo ter origem renal ou pararrenal. A aparência macroscópica é de uma massa nodular, bem circunscrita e encapsulada, ${ }^{6}$ com tamanho médio de $10 \mathrm{~cm} .{ }^{3}$ Contudo, relatos de casos mais recentes revelam uma diminuição de tamanho médio para $6,5 \mathrm{~cm},{ }^{5}$ permitindo abordagens terapêuticas poupadoras de néfrons.

Apesar do fato do Schwannoma normalmente ter comportamento benigno, a degeneração maligna pode ocorrer, trazendo consequências prognósticas ruins, ${ }^{4}$ especialmente no tocante à sobrevida livre de doença. A maioria dos pacientes diagnosticados com Schwannoma maligno morreram poucos meses após o tratamento cirúrgico. Apenas um caso relatado de malignidade permaneceu sem recidiva da lesão num período maior de 4 anos após a ressecção total, e sem necessitar de terapia adjuvante. $^{8}$

Diagnóstico pré-operatório de Schwannomas renais é difícil, pois a sintomatologia é vaga e os achados de imagem na tomografia computadorizada (TC) e ressonância nuclear magnética assemelham-se ao carcinoma de células renais (CCR), levando a erros de interpretação. Não é possível diferenciar radiologicamente tumores malignos de benignos. Frequentemente, os Schwannomas renais aparecem na TC como massas contrastadas, homogêneas e bem circunscritas, podendo haver áreas de degeneração cística, hemorragia ou calcificação, assim como acontece nos CCR. ${ }^{7}$ Achados na RNM podem dar algumas pistas, incluindo imagens com intensidade de isosinal em T1 e hipersinal em T2, ${ }^{1}$ com realce forte e homogêneo ao Gadolínio na parte sólida do tumor nas imagens em T1. Infelizmente, os achados radiológicos isolados não são suficientes para fazer o diagnóstico de Schwannoma Renal.

Acredita-se que a ressecção cirúrgica da lesão é o único tratamento efetivo, pois não se consegue diferenciar Schwannomas benignos de malignos antes da cirurgia, sendo necessário o estudo histopatológico para o diagnóstico definitivo. Além do que, esses tumores são frequentemente confundidos com CCR pelos exames de imagem, sendo a nefrectomia radical comumente indicada. Mas, a depender da localização e tamanho da lesão, a nefrectomia parcial é uma opção viável, bem como a cirurgia minimamente invasiva. O primeiro caso relatado de ressecção laparoscópica de um Schwannoma retroperitoneal ocorreu no ano de $1995 .{ }^{10} \mathrm{E}$ o primeiro relato de nefrectomia assistida por robô por um Schwannoma renal foi publicado em $2016 .^{5}$

Histologicamente, o tumor tem uma aparência bifásica característica, em que alterna áreas compactas de células fusiformes (padrão Antoni A) com áreas de células esparsas, sem uma ordem particular, com espaços microcísticos em um estroma mixoide (padrão Antoni B). ${ }^{1-13}$ Degeneração microcística com hemorragia e depósitos de hemossiderina associado com células de núcleo hipercromático são características degenerativas comuns em grandes Schwannomas usualmente encontrados no retroperitôneo. ${ }^{2}$ Schwannomas malignos foram confirmados histologicamente pela presença de mitoses, pleomorfismos e infiltração vascular. ${ }^{1}$

O diagnóstico definitivo de Schwannoma usualmente requer uma prova imuno-histoquímica de diferenciação neurogênica, utilizando a proteína S-100 como marcador. ${ }^{1-13}$ As células tumorais mostram uma imunorreatividade forte e difusa para S-100, além de forte imunorreatividade para o GFAP em áreas celulares. ${ }^{11}$

O prognóstico dos tumores de Schwannoma é bom, porém, a recorrência é possível. Dessa forma, seria necessário manter um seguimento pós-operatório. ${ }^{12}$ Recomenda-se exame de imagem após 6 meses para avaliar o status pós cirúrgico. ${ }^{8}$

\section{CONCLUSÃO}

Schwannomas renais, apesar de raros, merecem certa atenção no que diz respeito ao diagnóstico diferencial de lesões renais. Nefrectomia radical é normalmente recomendada, devido à dificuldade em determinar seu diagnóstico pré-operatório definitivo, além de haver a possibilidade de degeneração maligna do tumor. O diagnóstico histológico é frequentemente difícil e pode ser alcançado na maioria dos casos pela análise imunohistoquímica.

Apesar de se tratar de um tumor raro, não há estudos sobre ele sendo conduzidos. E compete à comunidade acadêmica relatar todos os casos, a fim de compartilhar seus resultados clínicos e de pesquisa, com o objetivo geral de melhora no cuidado ao paciente. $^{4}$ 


\section{REFERÊNCIAS}

1. Hung SF, Chung SD, Lai MK, Chueh SC, Yu HJ. Renal schwannoma: case report and literature review. Urology. 2008;72(3):716.e3-6.

2. Gobbo S, Eble JN, Huang J, Grignon DJ, Wang M, Martignoni G, et al. Schwan-noma of the Kidney. Mod Pathol. 2008;21(6):779-83.

3. Yang HJ, Lee MH, Kim DS, Lee HJ, Lee JH, Jeon YS. A case of renal schwan-noma. Korean J Urol. 2012;53(12):875-8.

4. Madueke I, Shore D. Robotic assisted laparoscopic excision of a renal schwanno-ma from a community hospital: a case report. Urology Case Reports. 2019;25:100891.

5. Kelley J, Collins R, Allam C. Robot-assisted laparoscopic renal schwannoma exci-sion. J Endourol Case Rep. 2016;2(1):221-3.

6. Iannaci G, Crispino M, Cifarelli P, Montella M, Panarese I, Ronchi A, et al. Epi-thelioid angiosarcoma arising in schwannoma of the kidney: report of first case and re-view of the literature. World J Surg Oncol. 2016;14:29.

7. Mikkikineni H, Thupili CR. Benign renal schwannoma. J Urol. 2013;189(1):317-8.
8. Herden J, Drebber U, Ural Y, Zimmer S, Wille S, Engelmann UH. Retroperitoneal schwannomas of renal and pararenal origin: presentation of two case reports. Rare Tu-mors. 2015;7(1):5616.

9. Verze P, Somma A, Imbimbo C, Mansueto G, Mirone V, Insabato L. Melanotic Schwannoma: a case of renal origin. Clin Genitourin Center. 2014;12(1):e37-41.

10. Hanashima F, Yanaihara H, Hayashi T, Kaguyama H, Teranishi Y, Sakamoto H, et al. Laparoscopic non-clamping tumor enucleation of renal hilum schwannoma in a single kidney: a case report. Urol Case Rep. 2015;3(6):211-4.

11. Hall SJ, Williams ST, Jackson TA, Lee AT, McCullock TA. Retroperitoneal gas-trointestinal type schwannoma presenting as a renal mass. Urol Case Rep. 2015;3(6):206-8.

12. Kumano Y, Kawahara T, Chiba S, Maeda Y, Ohtaka M, Kondo T, et al. Retroper-itoneal schwannoma in the renal hilum: a case report. Case Rep Oncol. 2015;8(3):394-8.

13. Wang Y, Zhu B. A Case of schwannoma in kidney. Quant Imaging Med Sure. 2013;3(3):180-1.

\section{Como citar:}

Souza SS, Dias BH, Leitão AS Júnior, Regadas RP, Cerqueira JB, Oliveira RR. Relato de caso: Schwannoma de origem renal. Rev Med UFC. 2021;61(1):1-4. 